\title{
Application of the crystalline sponge method for structure determination of persistent organic pollutants of agrochemical degradation products
}

\author{
T. Kikuchi ${ }^{1}$, K. Takagi $^{2}$, F. Sakakibara ${ }^{3}$, T. Sato ${ }^{1}$ \\ ${ }^{1}$ Rigaku Corporation, 3-9-12 Matsubara-cho, Akishima, Tokyo 196-8666, Japan, \\ ${ }^{2}$ National Agriculture and Food Research Organization, 3-1-3 Kannondai, Tsukuba-shi, Ibaraki 305-8604, Japan, \\ ${ }^{3}$ Sigma-Aldrich Japan, Arco Tower 5F, 1-8-1 Shimomeguro, Meguro-ku, Tokyo 153-8927.

$$
\text { ta-kiku@rigaku.co.jp }
$$

Identification of agrochemical degradation metabolites occurring in soil, water, and crops, and assessment of their toxicity are of great importance in view of food safety. A common approach to identify the structures of unknown metabolites is to synthesize their canditdates as reference standards based on the structures estimated from chemical information obtained from nuclear magnetic resonance (NMR) and mass spectrometric (MS) analyses, and to compare the unknown metabolites with the reference standards.

However, agrochemical degradation metabolites are usually obtained in only very small amounts, and a multitude of structures can occur in the environment and crops. Therefore, synthesizing all such potential metabolites and similar candidate compounds involves extremely time- and money-consuming efforts. If the molecular structures of a wide range of metabolites with very small amount can directly be determined by single crystal X-ray (SCX) analysis, the time and economic costs will dramatically be reduced.

The crystalline sponge (CS) method is a novel technique for sample preparation of SCX developed by Prof. Fujita.[1] The CS method utilizes a crystal of metal-organic framework (MOF) as a crystalline molecular container that can incorporate a wide variety of small molecules within the pores and arrange the molecules in a regular pattern according to the periodicity of the host crystal. As a result, the host-guest complex as a whole can be regarded as a single crystal.

The CS method is very advantageous for structure determination of the agrochemical degradation metabolites. Crystal preparation using one crystal of MOF enables trace analysis with a sub-microgram scale. As no other approach is able to produce accurate structural determinations with just micrograms of unidentified sample purified by preparative HPLC, based on degradation/metabolism experiments on a laboratory scale, SCX analysis combined with the CS method thus offers the potential of dramatically changing the conventional modality of degradation metabolite analysis.

In this presentation, we will show the initial examples of the structure identification of agrochemical metabolites (Fig. 1).
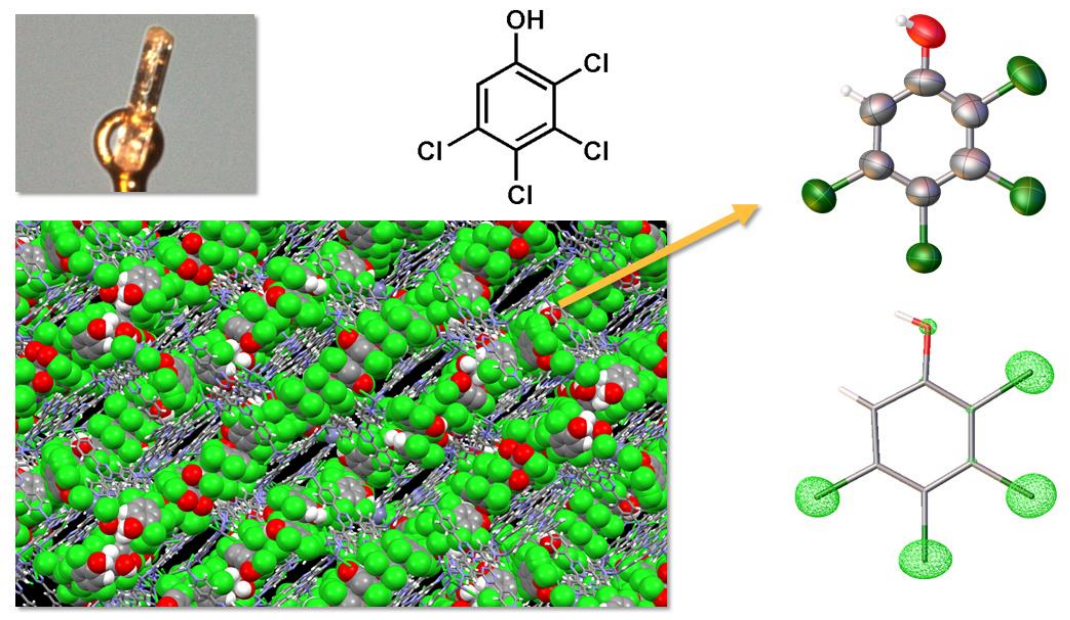

Figure 1. Structure identification of a polychlorine agrochemical metabolite by the CS method.

[1] Inokuma, Y., Yoshioka, S., Ariyoshi, J., Arita, T., Hirota, Y., Takada, K., Matsunaga, S., Rissanen, K. \& Fujita, M. (2013). Nature $495,461-467$.

Keywords: crystalline sponge method; agrochemical metabolites; trace analysis 